\title{
OPEN Disruption of Streptococcus mutans and Candida albicans synergy by a commensal streptococcus
}

\begin{abstract}
Joshua T. Huffines \& Jessica A. Scoffield ${ }^{\bowtie}$
Polymicrobial interactions in dental plaque play a significant role in dysbiosis and homeostasis in the oral cavity. In early childhood caries, Streptococcus mutans and Candida albicans are often co-isolated from carious lesions and associated with increased disease severity. Studies have demonstrated that metabolic and glucan-dependent synergism between $C$. albicans and $S$. mutans contribute to enhanced pathogenesis. However, it is unclear how oral commensals influence pathogen synergy. Streptococcus parasanguinis, a hydrogen peroxide $\left(\mathrm{H}_{2} \mathrm{O}_{2}\right)$ producing oral commensal, has antimicrobial activity against $S$. mutans. In this study, we utilized a three species biofilm model to understand the impact of S. parasanguinis on S. mutans and C. albicans synergy. We report that $S$. parasanguinis disrupts $S$. mutans and $C$. albicans biofilm synergy in a contact and $\mathrm{H}_{2} \mathrm{O}_{2}$-independent manner. Further, metabolomics analysis revealed a S. parasanguinis-driven alteration in sugar metabolism that restricts biofilm development by $S$. mutans. Moreover, $S$. parasanguinis inhibits $S$. mutans glucosyltransferase (GtfB) activity, which is important for glucan matrix development and GtfBmediated binding to $C$. albicans mannan. Taken together, our study describes a new antimicrobial role for S. parasanguinis and highlights how this abundant oral commensal may be utilized to attenuate pathogen synergism.
\end{abstract}

Early childhood caries (ECC) is a highly prevalent and costly biofilm-driven disease that disproportionately affects children of low socio-economic status ${ }^{1,2}$. Streptococcus mutans is commonly isolated from carious lesions of children with ECC, and as such, considered a primary etiological agent of dental caries. S. mutans is regarded as a highly cariogenic bacterium due to its ability to efficiently metabolize dietary carbohydrates into lactic acid, resist acid, and produce insoluble glucan, a sucrose-dependent exopolysaccharide (EPS) that is synthesized by glucosyltransferases (GTFs) and is a major virulence factor that permits the formation of robust biofilms and tooth adherence ${ }^{3-7}$. Although $S$. mutans is a primary cause of dental caries, oral plaque contains a consortium of microbes that interact synergistically or antagonistically ${ }^{8-12}$.

Candida albicans, an opportunistic fungal pathobiont residing throughout the body, is often co-isolated with $S$. mutans from carious lesions in children with severe ECC infections ${ }^{13-15}$. Several studies have reported mechanisms in which C. albicans and $S$. mutans display synergistic behavior. For example, $S$. mutans was found to significantly increase C. albicans colonization in a Drosophila melanogaster model. Moreover, co-infection with both species resulted in increased biofilm biomass and tooth decay compared to the single species infection in a rat caries model of infection ${ }^{11,16}$. Despite its involvement in ECC, C. albicans does not efficiently metabolize sucrose, but can readily utilize fructose and glucose $\mathrm{e}^{17,18}$. Further, GTFs have been shown to bind to C. albicans mannans, thus promoting EPS production and incorporation of the fungus into the biofilm ${ }^{19}$. In addition, exogenous GTFs promote $C$. albicans growth ${ }^{17}$. Synergism between $S$. mutans and C. albicans has been well-documen ted $^{11,16,17,19-21}$, however, the role of oral commensal streptococci in ECC microbial synergy is poorly understood.

Oral commensal streptococci are primary colonizers of the oral cavity and play a role in maintaining homeostasis by antagonizing oral pathogens via the production of hydrogen peroxide $\left(\mathrm{H}_{2} \mathrm{O}_{2}\right)$. Streptococcus parasanguinis, a mitis group streptococcus and one of the most abundant (5-40\% abundance) commensals on the tongue dorsum $^{22-25}$, exhibits enhanced biofilm formation in a $\mathrm{H}_{2} \mathrm{O}_{2}$-dependent manner during co-culture with Aggregatibacter actinomycetemcomitans while simultaneously inhibiting growth of this periopathogen ${ }^{26}$. Furthermore, $S$. parasanguinis reduces $S$. mutans biofilm formation and pathogenesis in a rat caries model of infection in a nitrite and $\mathrm{H}_{2} \mathrm{O}_{2}$-dependent manner ${ }^{27-29}$. Due to the remarkable ability of $S$. parasanguinis to inhibit both cariogenic and periodontal pathogens, we questioned whether $S$. parasanguinis could potentially disrupt $S$. mutans and $C$. albicans synergy. Although commensal streptococci have been shown to co-aggregate and form biofilms with 
A

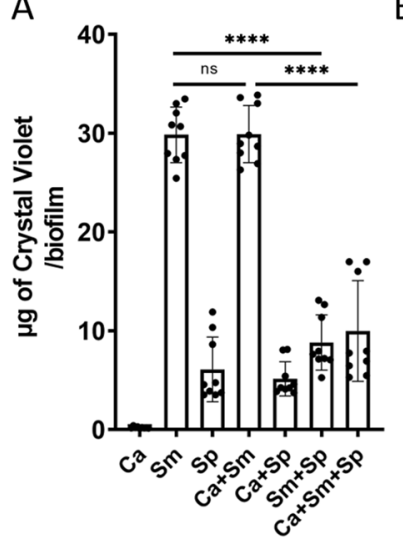

B

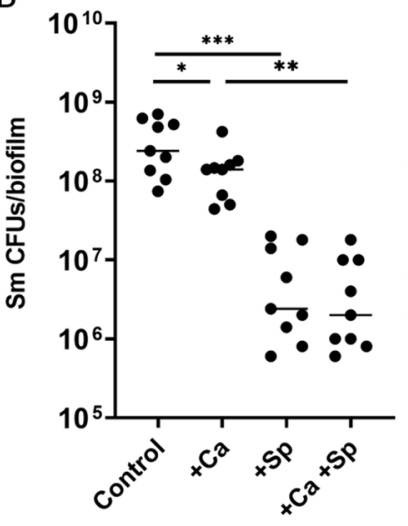

C

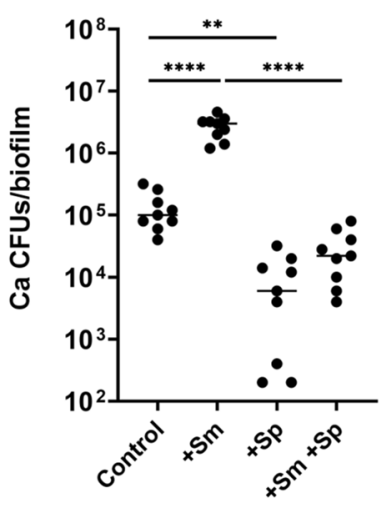

D

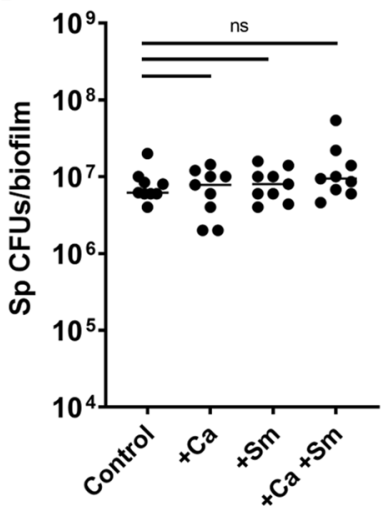

Figure 1. Streptococcus parasanguinis reduces S. mutans and C. albicans biofilm formation. (A) Crystal violet

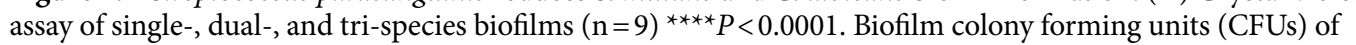
(B) S. mutans $(\mathrm{Sm}){ }^{\star} P=0.0448^{\star \star} P=0.0015^{\star * *} P=0.0007$ (C) C. albicans (Ca) ${ }^{\star \star} P=0.0012^{\star * * \star} P<0.0001$ (D) $S$. parasanguinis (Sp). All biofilms were grown in tryptic soy broth containing $0.5 \%$ yeast extract (TSBYE) and $1 \%$ sucrose for $16 \mathrm{~h}$ at $37^{\circ} \mathrm{C}$ with $5 \% \mathrm{CO}_{2}$. ns = not significant.

C. albicans $^{30}$, there are no studies that dissect how streptococci, like $S$. parasanguinis, modulate the interaction between $S$. mutans and C. albicans. In this study, we tested the role of S. parasanguinis in a three species biofilm model containing S. mutans and C. albicans. Here, we report that $S$. parasanguinis significantly reduces $S$. mutans and C. albicans biofilm synergy. Interestingly, the reduction in S. mutans and C. albicans biofilm formation was $\mathrm{H}_{2} \mathrm{O}_{2}$-independent, and was the result of a disruption in $S$. mutans sucrose metabolism and a shift in the global metabolic signature by $S$. parasanguinis. Lastly, we show that $S$. parasanguinis prevents $C$. albicans from adhering to glucan and inhibits the formation of glucan by blocking GTF activity. Taken together, our study reveals a new mechanism by which $S$. parasanguinis safeguards against ECC pathogens, specifically by blocking $S$. mutans sucrose utilization, and indicates that this commensal could potentially be used as a preventative measure for oral microbial diseases.

\section{Results}

Streptococcus parasanguinis inhibits $S$. mutans and C. albicans biofilm synergy. Streptococcus parasanguinis has been shown to inhibit the virulence of $S$. mutans, however, the role of this commensal streptococcus on S. mutans and C. albicans synergy is not clearly defined. In an effort to elucidate the interaction between $S$. parasanguinis and the ECC pathogens, S. mutans and C. albicans, we developed a three species biofilm model. In agreement with previous studies, crystal violet biomass quantification showed that $S$. parasanguinis and $C$. albicans produced relatively modest single species biofilms compared to the single species $S$. mutans biofilm (Fig. 1A). The dual S. parasanguinis and C. albicans biofilm was comparable to a single species $S$. parasanguinis biofilm. As expected, both the single $S$. mutans and dual S. mutans-C. albicans biofilms produced robust biofilms (Fig. 1A). Although previous studies report a synergistic increase in biofilm mass ${ }^{31}$, the dual-species biofilm between $C$. albicans and $S$. mutans was not significantly increased compared to the singlespecies $S$. mutans biofilm $(P>0.05$; Fig. 1A). Strikingly, the addition of $S$. parasanguinis resulted in a remarkable decrease in both two (S. mutans) and three species biofilms $(P<0.0001$; Fig. $1 \mathrm{~A})$. This result indicated the possibility that $S$. parasanguinis dominates the overall biofilm despite the presence of $S$. mutans and/or C. albicans. In order to quantify the abundance of each species within the biofilm we enumerated colony forming units (CFUs). Surprisingly, $S$. mutans CFUs were modestly decreased during co-culture with $C$. albicans $(P=0.0448$; Fig. 1B). Further, there was a 2-log reduction $(P=0.0007)$ in $S$. mutans CFUs in the presence of $S$. parasanguinis, regardless of whether C. albicans was present (Fig. 1B). In agreement with our previous study ${ }^{16}$, C. albicans CFUs were promoted $(\sim 1.5 \mathrm{log})$ in the dual biofilm with $S$. mutans $(P<0.0001$; Fig. 1C), but this was abolished when $S$. parasanguinis was added to the biofilm $(P<0.0001$; Fig. $1 C)$. Intriguingly, $S$. parasanguinis biomass did not change when co-cultured in single, dual, or three species biofilms (Fig. 1D). To gain insight into the overall structure of the multi-species biofilms, we used fluorescently labeled bacterial strains to visualize the biofilm architecture. Using GFP-labeled S. mutans, mCherry-labeled S. parasanguinis, and calcofluor white stained C. albicans, biofilm images were obtained using confocal laser scanning microscopy (CLSM). As expected, the presence of $S$. parasanguinis decreased the size of the $S$. mutans microcolonies in both the dual and three-species biofilms compared to the control biofilm (Fig. 2). Taken together, these data demonstrate S. parasanguinis inhibits not only $S$. mutans biofilm development, but restricts the incorporation of C. albicans into multi-species biofilms.

Inhibition of $S$. mutans biofilm is $\mathrm{H}_{2} \mathrm{O}_{2}$ and contact-independent. Oral commensal streptococci produce $\mathrm{H}_{2} \mathrm{O}_{2}$ to antagonize other bacteria ${ }^{28,32-35}$. The primary enzyme responsible for $\mathrm{H}_{2} \mathrm{O}_{2}$ production in $S$. parasanguinis is pyruvate oxidase, which is encoded by the pox $L$ gene $\mathrm{e}^{27,28,35,36}$. To investigate the potential mechanism of biofilm inhibition by $S$. parasanguinis and determine whether $\mathrm{H}_{2} \mathrm{O}_{2}$ production mediates this interference, both $S$. mutans and dual C. albicans-S. mutans biofilms were grown with wild-type $S$. parasanguinis or a 


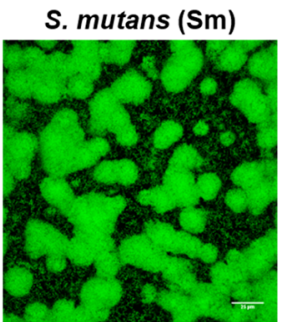

C. albicans (Ca)

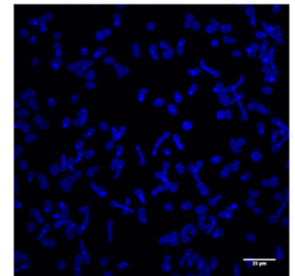

S. parasanguinis $(\mathrm{Sp})$

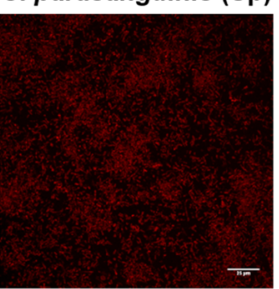

$\mathrm{Sm}+\mathrm{Ca}$

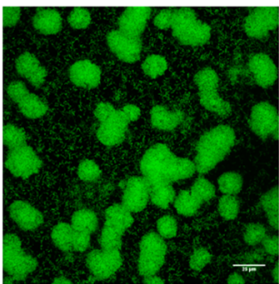

$\mathrm{Ca}+\mathrm{Sm}$

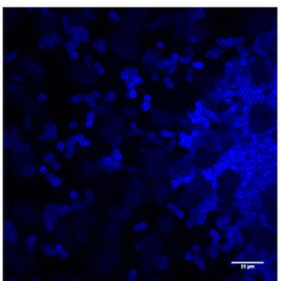

$\mathrm{Sp}+\mathrm{Ca}$

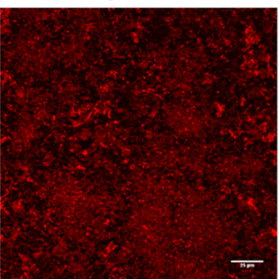

$S m+S p$

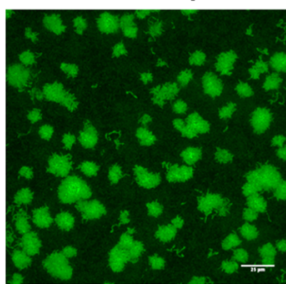

$\mathrm{Ca}+\mathrm{Sp}$

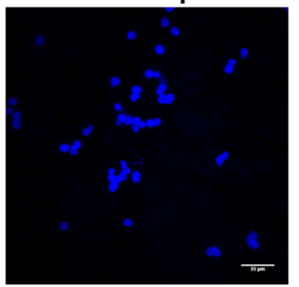

$S p+S m$

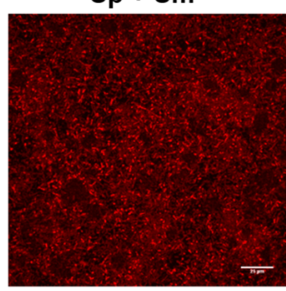

Three Species

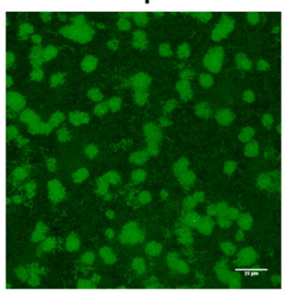

Three Species

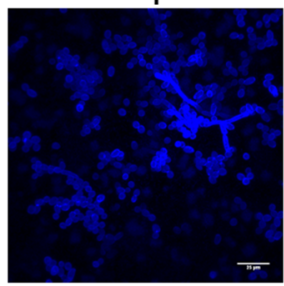

Three Species

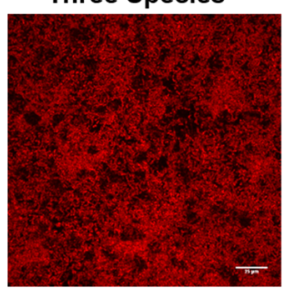

Figure 2. Streptococcus parasanguinis alters the structure of mixed biofilms. Confocal scanning laser microscopy images of wild-type S. mutans, C. albicans, and S. parasanguinis single and mixed biofilms at 60X magnification. S. mutans was labeled with green fluorescent protein (GFP), C. albicans was stained with calcofluor white, and S. parasanguinis was labeled with mCherry. All biofilms were grown in tryptic soy broth containing $0.5 \%$ yeast extract (TSBYE) and $1 \%$ sucrose for $16 \mathrm{~h}$ at $37^{\circ} \mathrm{C}$ with $5 \% \mathrm{CO}_{2}$. Scale bar: $25 \mu \mathrm{m}$. Images were acquired using the Nis Elements 5.0 Imaging Software (https://www.microscope.healthcare.nikon.com/ products/software/nis-elements).

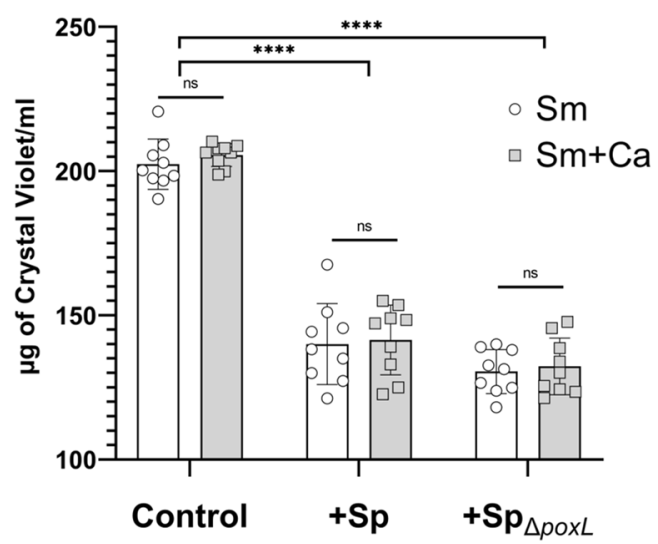

Figure 3. Inhibition of $S$. mutans single and dual biofilms with C. albicans by S. parasanguinis is contact and $\mathrm{H}_{2} \mathrm{O}_{2}$-independent. Quantification of single and dual species biofilms exposed to wild-type or pyruvate oxidase $\left(\right.$ poxL) deficient $S$. parasanguinis in transwells. ns = not significant; ${ }^{* * *} P<0.0001$. All biofilms were grown in tryptic soy broth containing $0.5 \%$ yeast extract (TSBYE) and $1 \%$ sucrose for $16 \mathrm{~h}$ at $37^{\circ} \mathrm{C}$ with $5 \% \mathrm{CO}_{2}$.

pyruvate oxidase deficient mutant $(\triangle p o x L)$ in transwell plates to physically separate $S$. parasanguinis from $S$. mutans and C. albicans. As shown in Fig. 3, the $S$. mutans single and dual (C. albicans) biofilms were reduced $(P<0.0001)$ at comparable levels by the presence of wild-type $S$. parasanguinis or the $\Delta$ poxL mutant in the transwell insert. These data indicate that the inhibition by $S$. parasanguinis was independent of physical contact and $\mathrm{H}_{2} \mathrm{O}_{2}$ production. 
A

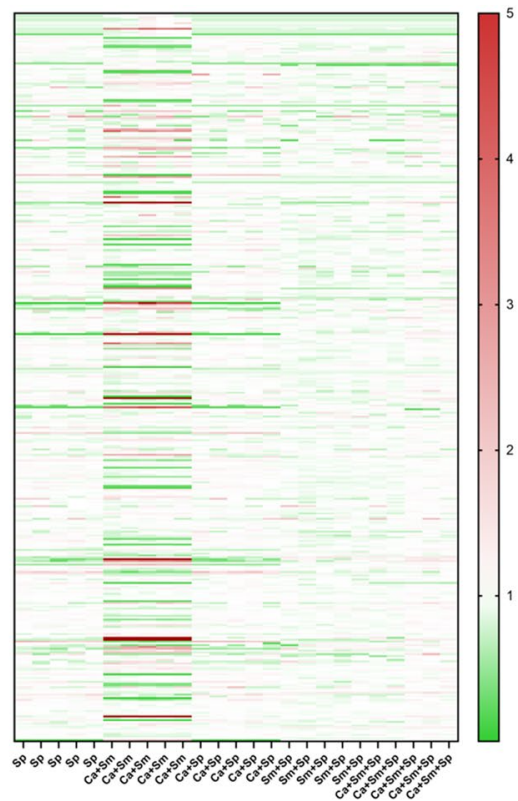

B

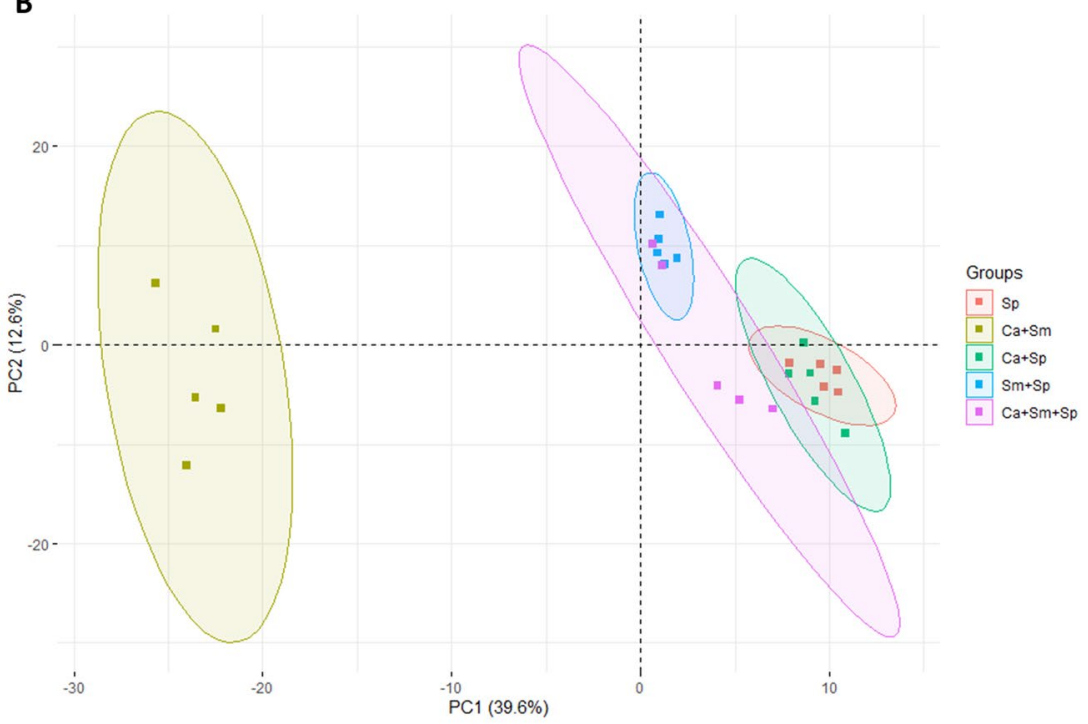

Figure 4. Streptococcus parasanguinis dominates the metabolic profile in dual- and tri-species cultures. (A) Global metabolomics profiling of dual- and tri-species cultures and single-species $S$. parasanguinis cultures. (B) Principle Component Analysis. Sp: S. parasanguinis; Sm: S. mutans; Ca: C. albicans. Metabolomics data were collected for 5 replicates in each group. All cultures were grown in tryptic soy broth containing $0.5 \%$ yeast extract (TSBYE) and $1 \%$ sucrose for $16 \mathrm{~h}$ at $37^{\circ} \mathrm{C}$ with $5 \% \mathrm{CO}_{2}$.

Metabolomics reveals $S$. parasanguinis-altered sugar metabolism in multi-species biofilms. Streptococcus mutans biofilm development is driven by carbohydrate metabolism and the synthesis of a glucan matrix from sucrose $\mathrm{e}^{3-5,11,17,37}$. To probe how $S$. parasanguinis impacts sugar metabolism, we conducted global, untargeted metabolic profiling on dual- and three-species combined planktonic and biofilm cultures as well as single species $S$. parasanguinis cultures to delineate how $S$. parasanguinis modulates the multi-species biofilm metabolome (Fig. 4A). Principle component analysis revealed the dual C. albicans-S. mutans group to be vastly different than biofilms cultured with S. parasanguinis, with PC1 accounting for $39.6 \%$ of the variance (Fig. 4B). Shifts in the metabolic signatures of dual and three species biofilms were largely driven by S. parasanguinis, as the metabolome profiles of multi-species cultures closely resembled a single species $S$. parasanguinis biofilm. The dual C. albicans and S. mutans biofilm revealed an enriched presence of broad classes of metabolites, including lipids, peptides, cofactors, and amino acids compared to single or dual species biofilms containing $S$. parasanguinis, including compounds such as tyrosol and glycerol, both of which are indicated in biofilm development for C. albicans ${ }^{38,39}$ (Figs. S1-S4). Further, the dual C. albicans and S. mutans biofilms had elevated concentrations of sugar alcohols, fructose, glucose, and fructose compared to single and dual species biofilms containing S. parasanguinis, (Fig. 5A), suggesting that $S$. parasanguinis potentially consumes these sugars. Notably, sucrose consumption was drastically reduced in all biofilms containing $S$. parasanguinis compared to the dual-species S. mutans-C. albicans biofilm. (Fig. 5A).

To confirm the altered sucrose metabolism, we quantified the sucrose concentrations from biofilm supernatants of single, dual, and three-species cultures (Fig. 5B). Compared to the un-inoculated media control, $S$. parasanguinis utilized a small fraction of sucrose, $S$. mutans single and dual biofilms with C. albicans utilized all of the sucrose, but C. albicans did not utilize sucrose when grown alone, as previously reported ${ }^{17}$. There was no significant difference between a single species $S$. parasanguinis or dual culture with $C$. albicans (Fig. 5B), suggesting that any sucrose consumption in these dual biofilms is solely attributed to the presence of $S$. parasanguinis. In agreement with our metabolomics findings, $S$. parasanguinis reduced sucrose utilization in the dual (S. mutans) and three-species biofilm cultures $(P<0.0001)$. Overall, these data show that $S$. parasanguinis drives metabolism in polymicrobial cultures and may restrict $S$. mutans and $C$. albicans synergy by blocking sucrose utilization.

Streptococcus parasanguinis blocks $C$. albicans glucan binding and impairs $S$. mutans GTF activity. GtfB-mediated glucan production and binding to C. albicans mannan is considered to be a central mechanism of synergy between the two species ${ }^{17,19}$. Therefore, we tested whether S. parasanguinis diminishes the ability of $C$. albicans to adhere and form biofilms with glucan that is synthesized from cell-free $S$. mutans GTFs. First, to confirm that a single C. albicans biofilm is indeed promoted by the addition of exogenous GTFs from $S$. mutans, we cultured C. albicans with ethanol precipitated supernatants from wild-type and gtfB deficient $S$. mutans cultures. As shown in Fig. 6A, the addition of $25 \mu \mathrm{L}$ supernatant from wild-type $S$. mutans increased the $C$. albicans biofilm by approximately 3.5 fold compared to the $C$. albicans control $(P<0.0001)$. Supernatant harvested from the $g t f B$ mutant showed defects in the ability to promote $C$. albicans biofilm compared to wildtype $S$. mutans. Next, we wanted to determine if $S$. parasanguinis blocks the ability of GTFs to enhance the $C$. 
A

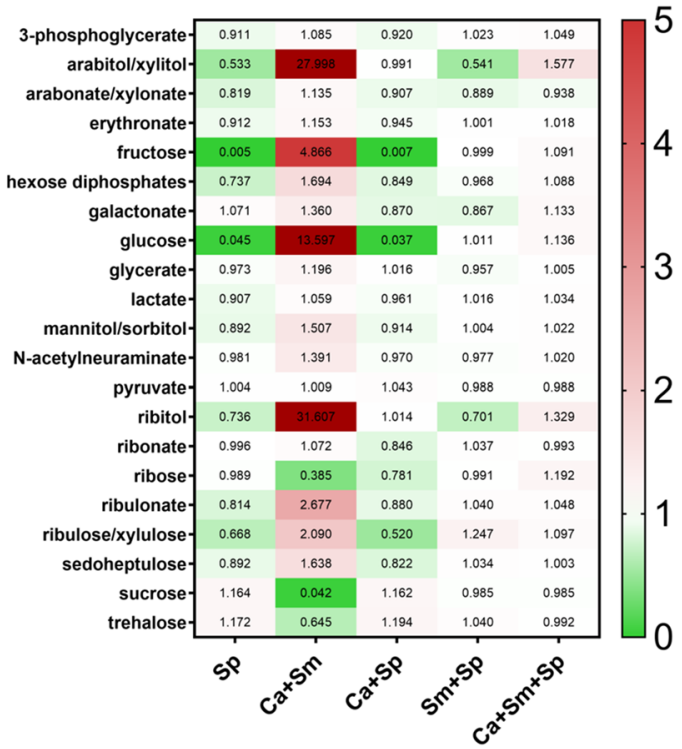

B 35

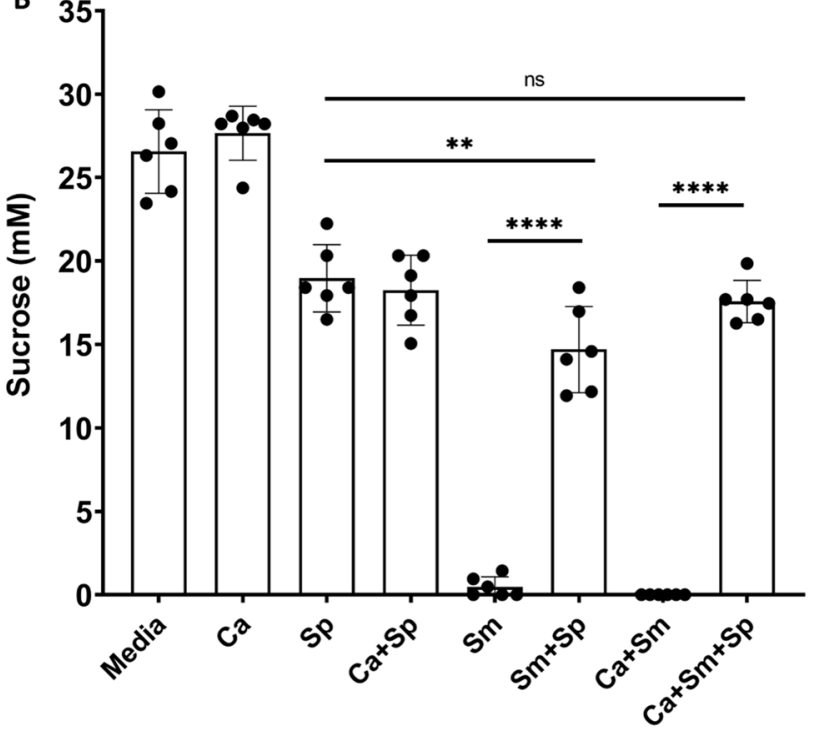

Figure 5. Presence of $S$. parasanguinis reduces sucrose utilization by $S$. mutans (A) Heatmap of carbohydrate metabolites (Value is median normalized) (B) Sucrose assay of single-, dual-, and tri-species cultures. Sp: $S$. parasanguinis; Sm: S. mutans; Ca: C. albicans. Metabolomics data were collected for 5 replicates in each group. ns $=$ not significant; ${ }^{* *} P=0.0096 ;{ }^{* * *} P<0.0001$. All cultures were grown in tryptic soy broth containing $0.5 \%$ yeast extract (TSBYE) and $1 \%$ sucrose for $16 \mathrm{~h}$ at $37^{\circ} \mathrm{C}$ with $5 \% \mathrm{CO}_{2}$.

A

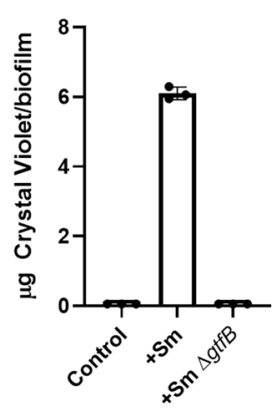

B

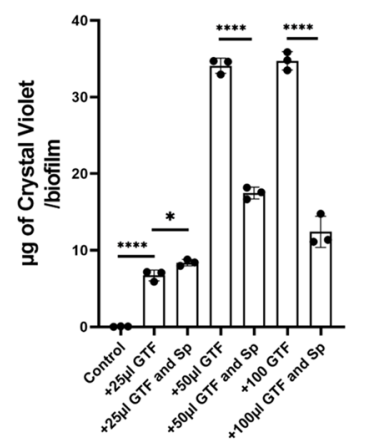

C C. albicans

C. albicans + GTFs

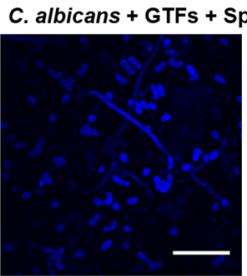

Figure 6. Streptococcus parasanguinis blocks synergy between GTFs and C. albicans. (A) Crystal violet assay of $C$. albicans biofilms with supernatant from wild-type or the gtfB mutant strain of $S$. mutans. (B) Crystal violet assay of $C$. albicans and C. albicans-S. parasanguinis biofilm with purified GTFs from overnight $S$. mutans cultures. ${ }^{\star} P=0.0246{ }^{\star * * \star} P<0.0001$; (C) Confocal laser scanning microscopy images of $C$. albicans biofilm stained with calcofluor white with GTFs or GTFs and $S$. parasanguinis. Scale bar: $50 \mu \mathrm{m}$. All cultures were grown in tryptic soy broth containing $0.5 \%$ yeast extract (TSBYE) and $1 \%$ sucrose for $16 \mathrm{~h}$ at $37^{\circ} \mathrm{C}$ with $5 \%$ $\mathrm{CO}_{2}$.

albicans biofilm. To test this we added varying concentrations $(25,50$, and $100 \mu \mathrm{L})$ of GTF supernatant to single C. albicans biofilms (+/-S. parasanguinis). As shown in Fig. 6B, the addition of all concentrations significantly increased $C$. albicans biofilm, but $S$. parasanguinis dramatically reduced the ability of $C$. albicans to increase its biofilm for both the $50 \mu \mathrm{L}$ and $100 \mu \mathrm{L}$ groups $(P<0.0001)$. Finally, to verify this finding, we visualized C. albicans using calcofluor white and CLSM in biofilms containing $100 \mu \mathrm{L}$ of GTFs and S. parasanguinis. Imaging con- 
A

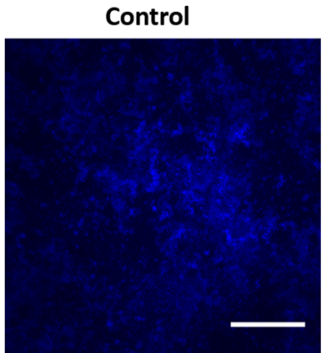

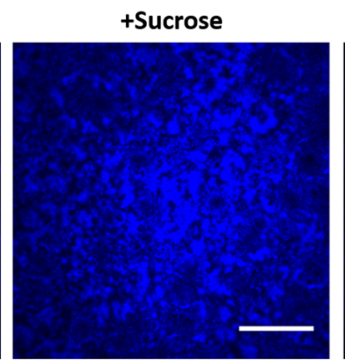

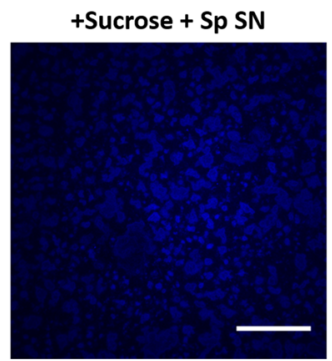

B

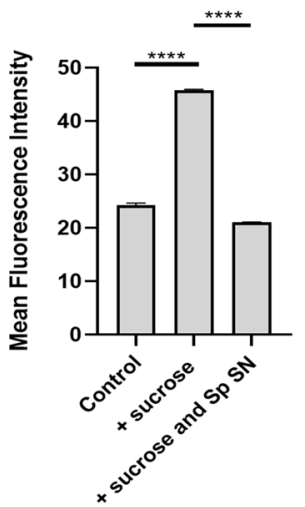

Figure 7. Streptococcus parasanguinis blocks glucan formation by GTFs. Confocal laser scanning microscopy of purified GTFs from $S$. mutans culture and cascade Blue-labeled dextran in (A) TSBYE, TSBYE $+1 \%$ Sucrose, or TSBYE $+1 \%$ sucrose with $100 \mu \mathrm{L}$ of $S$. parasanguinis supernatant (SN). (B) Fluorescence intensity of each biofilm. ${ }^{* * *} \mathrm{P}<0.0001$ Scale bar: $50 \mu \mathrm{m}$. All cultures were grown for $16 \mathrm{~h}$ at $37^{\circ} \mathrm{C}$ with $5 \% \mathrm{CO}_{2}$.

firmed that $S$. mutans supernatant containing exogenous GTFs do indeed promote the C. albicans biofilm, and this promotion is ablated by $S$. parasanguinis (Fig. 6C).

Due to the ability of $S$. parasanguinis to induce dramatic shifts in $S$. mutans sugar utilization, particularly sucrose, and inhibit C. albicans from forming optimal biofilms with GTFs, we reasoned that this result could potentially be due to impaired GTF activity by $S$. parasanguinis since GTFs convert sucrose to glucan. It is well established that glucan plays a critical role in the formation of a mature $S$. mutans biofilm and the establishment of mixed species biofilms $s^{37,40}$. To investigate whether altered sucrose utilization was driven by a reduction in GTF activity by $S$. parasanguinis we purified GTFs from the supernatant of $S$. mutans cultures. Using a Cascade blue glucan probe, we used confocal microscopy to detect modifications in the ability of purified GTFs to synthesize glucan from sucrose when S. parasanguinis was present. In our positive control, purified GTFs produced copious amounts of glucan with the addition of sucrose compared to GTFs that received no sucrose (negative control) (Fig. 7A). Although GTFs that did not receive sucrose produced a marginal amount of glucan compared to our positive control, we suspect this minor increase was due to carbohydrates in the growth media that permitted a small amount of glucan to be synthesized by purified GTFs. Remarkably, the addition of S. parasanguinisconditioned, filtered-sterilized supernatant dramatically reduced the formation of glucan, therefore resulting in a drop in fluorescence intensity (Fig. 7A,B). S. parasanguinis also decreased glucan formation in single and mixed species biofilms containing $S$. mutans (Fig. S5). Overall, these data demonstrate that $S$. parasanguinis not only shifts carbohydrate metabolism during multi-species biofilm development, but can directly inhibit GTF activity through an unknown secreted factor.

\section{Discussion}

Early childhood caries is a biofilm driven disease with $S$. mutans and C. albicans playing a considerable role $e^{9,10,13,15}$. Previous studies have demonstrated synergistic activity to either $S$. mutans or C. albicans when cultured together ${ }^{11,17,19-21,40}$. In addition, C. albicans and oral commensal streptococci have been shown to physically interact and display synergistic behavior within biofilms ${ }^{30,41}$, however, the impact of oral commensal streptococci in the $S$. mutans-C. albicans interaction is unclear. In this study, we demonstrate that $S$. parasanguinis, one of the most prominent commensals in the oral cavity ${ }^{22,23}$, interferes with $S$. mutans and C. albicans biofilm synergy in a $\mathrm{H}_{2} \mathrm{O}_{2}$ and contact independent manner. Supporting this observation, CLSM analysis revealed a reduction in the size of $S$. mutans microcolonies in the presence of $S$. parasanguinis. Remarkably, S. parasanguinis altered the global metabolic signature of the $S$. mutans-C. albicans cultures. Further, we provide evidence that S. parasanguinis blocks C. albicans from binding glucan, presumably by directly restricting GTF activity, which is essential for $C$. albicans binding and incorporation into the $S$. mutans biofilm, as well as robust S. mutans biofilm development. Numerous studies have reported that $\mathrm{H}_{2} \mathrm{O}_{2}$ production by commensal streptococci is the main virulence determinant that antagonizes $S$. mutans ${ }^{28,35,42}$. Paradoxically, we found that $\mathrm{H}_{2} \mathrm{O}_{2}$ had no role in the interference of $S$. mutans-C. albicans biofilm synergy as the level of biofilm inhibition by $\mathrm{H}_{2} \mathrm{O}_{2}$-deficient $S$. parasanguinis was comparable to wild-type. Overall, our studies highlight a new antimicrobial function for $S$. parasanguinis and illustrates how this commensal may potentially be used to target $S$. mutans glucan production, which is critical for not only $S$. mutans pathogenesis, but synergism with C. albicans.

Our global metabolomics analysis revealed that $S$. parasanguinis dominated the metabolic profile of dual and tri-species biofilms. Sucrose has consistently been recognized as the primary substrate that governs $S$. mutans virulence due to the ability of GTFs to synthesize glucan and establish a resilient biofilm ${ }^{3-6,8}$. C. albicans does not efficiently metabolize sucrose, but instead metabolizes intermediates released from the breakdown of sucrose by $S$. mutans, such as fructose and glucose $\mathrm{e}^{43}$. In our study, fructose and glucose concentrations were elevated in the dual S. mutans and C. albicans biofilm, but the addition of S. parasanguinis severely reduced the levels of these substrates. Fructose, glucose, and sucrose are critical sugars in a recently proposed model of synergism between S. mutans and C. albicans ${ }^{17}$. Tyrosol, known for stimulating hyphae formation in C. albicans ${ }^{38}$, was found to be 
elevated in the C. albicans-S. mutans culture, but was largely absent from biofilms containing S. parasanguinis even though we observed more hyphae formation in the three species biofilms and in single species C. albicans cultures that were incubated with cell-free, purified GTFs. Although it is unclear as to how hyphae formation impacts synergistic biofilm formation, the GTF-dependent synergism is independent of hyphae formation ${ }^{19}$. Glycerol, indicated as an important regulator for biofilm formation by C. albicans ${ }^{39}$, was also higher in the dual species biofilm with C. albicans and S. mutans. One limitation of our study is that the metabolomics findings are representative of both planktonic and biofilm cultures. Although the majority of $S$. mutans cells commit to biofilm formation in the presence of sucrose rather than a planktonic mode of growth, understanding how commensals impact the metabolome of planktonic versus biofilm cells in mixed cultures would be beneficial. It is also important to note that the reduction in sugar metabolism cannot be completely explained by a reduction in S. mutans CFUs. S. parasanguinis reduces $S$. mutans CFUs by approximately 2-log. However, S. parasanguinis reduced $S$. mutans sucrose consumption by approximately 15 -fold and inhibited the ability of GTFs to utilize sucrose in the cell-free assay when there were no $S$. mutans cells present. Overall, our findings indicate that $S$. parasanguinis may consume substrates that are essential for pathogen synergy and possibly sequester nutrients from C. albicans, thus making this fungus less competitive in a polymicrobial environment where it is unable to take advantage of cross-feeding from $S$. mutans.

A surprising result in our study was that $S$. parasanguinis inhibited the ability of GTFs to synthesize glucan, which could explain why $C$. albicans biofilm formation was reduced when this organism was cultured with GTF supernatant and the commensal. Alternatively, since C. albicans has been shown to coaggregate with oral streptococci ${ }^{30,41}, S$. parasanguinis may also obstruct the ability of GTFs to access mannans on the C. albicans cell surface. It is unlikely that $S$. parasanguinis and C. albicans compete for GTFs or glucan binding. Although S. parasanguinis surface adhesins have been shown to enhance biofilm formation by binding to biofilm matrix polysaccharides produced by Pseudomonas aeruginos $a^{44}$, there is no evidence that demonstrate that exogenous GTFs can promote $S$. parasanguinis biofilm formation. Given that GTFs, particularly GtfB, are critical for biofilm formation, adherence of $S$. mutans to the tooth surface, and C. albicans-S. mutans synergy, the development of $\mathrm{GtfB}$ inhibitors is viewed as a viable strategy to target $S$. mutans pathogenesis. Structure-based screening has identified small molecules that have demonstrated efficacy against GTF activity ${ }^{45-47}$. Ideally, GTF targets should display selective activity against $S$. mutans, but not disturb the composition or function of the oral microbiota, especially commensals. Our findings suggest that commensal-mediated inhibition of $S$. mutans GTF activity may serve as a novel strategy to hinder $S$. mutans pathogenesis while preserving the integrity of the oral microbiome. Further dissection of the exact mechanism or molecule (s) involved in S. parasanguinis-mediated inhibition of $S$. mutans GTF activity is warranted and will be the focus of future studies.

To our knowledge, S. parasanguinis is the only oral commensal that has demonstrated broad antimicrobial and anti-biofilm effects against diverse pathogens, including cariogenic, periodontal, and respiratory pathogens. For example, the $S$. parasanguinis biofilm is promoted in a $\mathrm{H}_{2} \mathrm{O}_{2}$-dependent manner during co-culture with the periopathogen A. actinomycetemcomitans, yet this pathogen loses viability in this model ${ }^{26}$. In addition, $S$. parasanguinis has been shown to inhibit $S$. mutans pathogenesis in $\mathrm{H}_{2} \mathrm{O}_{2}$ and nitrite-dependent manner in a rat caries model of infection ${ }^{42}$. Moreover, the translocation of $S$. parasanguinis into the cystic fibrosis (CF) lung has been associated with improved lung function in CF patients ${ }^{48}$, presumably by antagonizing the major CF pathogen $P$. aeruginosa. S. parasanguinis reduces viability and pathogenesis of the respiratory pathogen $P$. aeruginosa in vitro and in a $D$. melanogaster infection model via reactive nitrogenous intermediate production ${ }^{34}$. Lastly, a $S$. parasanguinis surface adhesin (BapA1) facilitates the enhancement of commensal biofilm formation while $P$. aeruginosa biofilm development is restricted during co-culture ${ }^{44}$. Taken together, these studies exhibit that the oral commensal S. parasanguinis has the potential to be used as a therapeutic to combat a variety of microbial infections.

In summary, this study provides new insight into how $S$. parasanguinis disrupts synergy between $S$. mutans and C. albicans by interfering with GTF activity. The observation that $S$. parasanguinis hinders GTF activity requires future investigation to identify the specific molecule (s) that mediates this inhibition and test whether it can be harnessed for the development of improved therapeutics. Our present study, as well as previous studies, signifies that $S$. parasanguinis is a unique commensal that may be suitable for prebiotic or probiotic use. Future studies that explore the molecular mechanisms of $S$. parasanguinis microbial antagonism will strengthen our understanding of the complexities involved in polymicrobial relationships and aid in the development commensal-derived therapies that are active against oral infections.

\section{Materials and methods}

Microbial strains and growth conditions. C. albicans SC5314, S. mutans UA159, S mutans $\Delta$ gtfB, S. parasanguinis FW213, and S. parasanguinis $\Delta$ pox $L^{34}$ strains were used in this study. SC5314 was grown in Yeast Peptone Dextrose (YPD) whereas UA159 and FW213 were grown in Tryptic Soy Broth + 5\% (w/v) Yeast Extract (TSBYE).

Biofilm and transwell assays. Overnight cultures were grown to mid-exponential phase $\left(\mathrm{OD}_{600}=0.5\right)$ and approximately $1 \times 10^{4}$ colony forming units per milliliter $(\mathrm{CFU} / \mathrm{mL})$ were seeded into the biofilm for each species. All single, dual, and three-species biofilms were grown in TSBYE $+1 \%(\mathrm{w} / \mathrm{v})$ sucrose for $16 \mathrm{~h}$ at $37^{\circ} \mathrm{C}+5 \%$ $\mathrm{CO}_{2}$. For all transwell experiments, a 6 -well transwell insert system with a $0.4 \mu \mathrm{m}$ pore polycarbonate membrane (Corning) was used to grow S. parasanguinis and the poxL mutant in the upper chamber and S. mutans and C. albicans biofilms in the lower well and incubated as described above. Microtiter plates were gently washed 2 times, blotted, and stained with $0.1 \%$ crystal violet. Following crystal violet staining, biofilms were washed, dried, and dissolved with $30 \%$ acetic acid as described previously ${ }^{49}$. To quantify biofilms to obtain values in the 
linear range of the Biotek plate reader, a standard curve was generated by serially diluting acetic acid dissolved biofilms and measuring the $\mathrm{OD}_{562}$ for each dilution. Using a standard curve, the $\mu \mathrm{g}$ of crystal violet was calculated using a trendline of the linear portion $\left(\mathrm{R}^{2}=0.9966\right)$. All dilutions were measured in 96 -well plates. Each assay was performed in triplicate wells and repeated three times.

Quantification of colony forming units (CFUs). All biofilms were gently washed with sterile PBS twice before adding $200 \mu \mathrm{L}$ of sterile PBS for plating. The biofilms were scraped up with a $200 \mu \mathrm{L}$ tip, vortexed for $10 \mathrm{~s}$, and were serially diluted. All dilutions were plated on Todd-Hewitt Broth or blood agar plates and were incubated at $37^{\circ} \mathrm{C}+5 \% \mathrm{CO}_{2}$ for a minimum of $16 \mathrm{~h}$ before counting.

Confocal laser scanning microscopy (CLSM). GFP-labeled UA159 and mCherry-labeled FW213 were used to visualize biofilms. All biofilms for confocal were grown in Ibidi $\mu$-Slide 8 well slides (Cat \#: 80826). Wells were gently washed with phosphate-buffered saline (PBS). Wells with $C$. albicans were stained with calcofluor white in PBS for 15 min before imaging. Biofilms were visualized on a Nikon A1 + confocal laser scanning microscope (CLSM) (Nikon Instruments Inc.) using a $60 \times$ oil immersion lens. Three dimensional biofilm images were acquired using the Nis Elements 5.0 Imaging Software. All images are representative of biofilms from 3 independent experiments.

Metabolomics analysis and sucrose quantification. Single, dual, and three species cultures were grown overnight in $20 \mathrm{~mL}$ of TSBYE $+1 \%$ sucrose in $50 \mathrm{~mL}$ conical tubes. Cells from the entire culture (planktonic and biofilm) were harvested to ensure at least $100 \mu \mathrm{L}$ of packed cells were available for each of the 5 replicates to meet Metabolon's processing criteria. Samples were stored in the $-80^{\circ} \mathrm{C}$ freezer prior to shipment on dry ice to Metabolon, Inc (Durham, NC). All samples were processed and analyzed for raw counts by Metabolon using liquid chromatography-mass spectrometry (LCMS) methods optimized for positive ions, negative ions, and polar compounds. Raw counts provided by Metabolon were median scaled with missing values imputed with the lowest value. Principal component analysis (PCA) was calculated and graphed using R. Heatmaps of the median scaled data was made using GraphPad Prism.

To measure sucrose concentrations single, dual, and three species cultures were grown and harvested as described above for the metabolomics samples. Samples were stored overnight at $-80^{\circ} \mathrm{C}$ prior to quantification. Sucrose concentrations were measured using the Glucose and Sucrose Assay Kit (Sigma Aldrich; Cat No. MAK013).

GTF precipitation, cell-free glucan formation, and glucan quantification. S. mutans proteins from overnight culture supernatants were ethanol precipitated at a 1:1 ratio of $100 \%$ ethanol to precipitate extracellular S. mutans GTFs. The ethanol/proteins were incubated at $-80^{\circ} \mathrm{C}$ for $1 \mathrm{~h}$, pelleted, and re-suspended in fresh TYE ( $\pm 1 \%$ sucrose). To test the role of S. parasanguinis supernatant on S. mutans glucan formation, $100 \mu \mathrm{L}$ of cell-free GTFs were added to $1 \mathrm{~mL}$ of TSBYE media that contained no sucrose, $1 \%$ sucrose, or $1 \%$ sucrose with the additional of $100 \mu \mathrm{L}$ of filtered-sterilized spent $S$. parasanguinis media from overnight cultures. Samples were dispensed in polystyrene dishes and incubated for $16 \mathrm{~h}$ at $37^{\circ} \mathrm{C}+5 \% \mathrm{CO}_{2}$ to permit glucan formation. For all experiments, $1 \mu \mathrm{M}$ dextran-conjugated Cascade Blue (Molecular Probes, Invitrogen) was added to the media before overnight incubation. Fluorescence was quantified using ImageJ.

Statistical analysis. For crystal violet, colony-forming units, and sucrose assays, we analyzed the data using Prism version 8.4.3 (GraphPad Software, LLC). An alpha value of 0.05 was used to determine statistical significance. For the metabolomics data that consisted of 5 replicates, one-way ANOVA was used to determine $P$ values for the single-species group and a two-way ANOVA was used for groups with multiple species.

Received: 28 July 2020; Accepted: 2 November 2020

Published online: 12 November 2020

\section{References}

1. Dye, B. A., Li, X. \& Thorton-Evans, G. Oral Health Disparities as Determined by Selected Healthy People 2020 Oral Health Objectives for the United States, 2009-2010. NCHS Data Brief 1-8 (National Center for Health Statistics, Hyattsville, 2012).

2. Casamassimo, P. S., Thikkurissy, S., Edelstein, B. L. \& Maiorini, E. Beyond the dmft: The human and economic cost of early childhood caries. J. Am. Dent. Assoc. 140, 650-657. https://doi.org/10.14219/jada.archive.2009.0250 (2009).

3. Aoki, H., Shiroza, T., Hayakawa, M., Sato, S. \& Kuramitsu, H. K. Cloning of a Streptococcus mutans glucosyltransferase gene coding for insoluble glucan synthesis. Infect. Immun. 53, 587-594. https://doi.org/10.1128/IAI.53.3.587-594.1986 (1986).

4. Hanada, N. \& Kuramitsu, H. K. Isolation and characterization of the Streptococcus mutans gtfC gene, coding for synthesis of both soluble and insoluble glucans. Infect. Immun. 56, 1999-2005. https://doi.org/10.1128/IAI.56.8.1999-2005.1988 (1988).

5. Hanada, N. \& Kuramitsu, H. K. Isolation and characterization of the Streptococcus mutans gtfD gene, coding for primer-dependent soluble glucan synthesis. Infect. Immun. 57, 2079-2085. https://doi.org/10.1128/IAI.57.7.2079-2085.1989 (1989).

6. Bowen, W. H. \& Koo, H. Biology of Streptococcus mutans-derived glucosyltransferases: Role in extracellular matrix formation of cariogenic biofilms. Caries Res. 45, 69-86. https://doi.org/10.1159/000324598 (2011).

7. Lemos, J. A. et al. The biology of Streptococcus mutans. Microbiol. Spectr. https://doi.org/10.1128/microbiolspec.GPP3-0051-2018 (2019).

8. Van Houte, J., Sansone, C., Joshipura, K. \& Kent, R. Mutans streptococci and non-mutans streptococci acidogenic at low pH, and in vitro acidogenic potential of dental plaque in two different areas of the human dentition. J. Dent. Res. 70, 1503-1507. https:// doi.org/10.1177/00220345910700120601 (1991). 
9. Palmer, C. A. et al. Diet and caries-associated bacteria in severe early childhood caries. J. Dent. Res. 89, 1224-1229. https://doi. org/10.1177/0022034510376543 (2010).

10. Kanasi, E. et al. Microbial risk markers for childhood caries in pediatricians' offices. J. Dent. Res. 89, 378-383. https://doi. org $/ 10.1177 / 0022034509360010(2010)$.

11. Falsetta, M. L. et al. Symbiotic relationship between Streptococcus mutans and Candida albicans synergizes virulence of plaque biofilms in vivo. Infect. Immun. 82, 1968-1981. https://doi.org/10.1128/IAI.00087-14 (2014).

12. Richards, V. P. et al. Microbiomes of site-specific dental plaques from children with different caries status. Infect. Immun. 85, e00106-00117. https://doi.org/10.1128/IAI.00106-17 (2017).

13. de Carvalho, F. G., Silva, D. S., Hebling, J., Spolidorio, L. C. \& Spolidorio, D. M. Presence of mutans streptococci and Candida spp. in dental plaque/dentine of carious teeth and early childhood caries. Arch. Oral. Biol. 51, 1024-1028. https://doi.org/10.1016/j. archoralbio.2006.06.001 (2006).

14. Raja, M., Hannan, A. \& Ali, K. Association of oral candidal carriage with dental caries in children. Caries Res. 44, 272-276. https ://doi.org/10.1159/000314675 (2010)

15. Bachtiar, E. W. \& Bachtiar, B. M. Relationship between Candida albicans and Streptococcus mutans in early childhood caries, evaluated by quantitative PCR. F1000Research 7, 1645. https://doi.org/10.12688/f1000research.16275.2 (2018).

16. Yang, C. et al. Antigen I/II mediates interactions between Streptococcus mutans and Candida albicans. Mol. Oral. Microbiol. 33, 283-291. https://doi.org/10.1111/omi.12223 (2018).

17. Ellepola, K. et al. Multi-omics analyses reveal synergistic carbohydrate metabolism in Streptococcus mutans-Candida albicans mixed-species biofilms. Infect. Immun. https://doi.org/10.1128/IAI.00339-19 (2019).

18. Williamson, P. R., Huber, M. A. \& Bennett, J. E. Role of maltase in the utilization of sucrose by Candida albicans. Biochem. J. 291(Pt 3), 765-771. https://doi.org/10.1042/bj2910765 (1993).

19. Hwang, G. et al. Candida albicans mannans mediate Streptococcus mutans exoenzyme GtfB binding to modulate cross-kingdom biofilm development in vivo. PLoS Pathog. 13, e1006407. https://doi.org/10.1371/journal.ppat.1006407 (2017).

20. Kim, D. et al. Candida albicans stimulates Streptococcus mutans microcolony development via cross-kingdom biofilm-derived metabolites. Sci. Rep. 7, 41332. https://doi.org/10.1038/srep41332 (2017).

21. He, J. et al. RNA-Seq reveals enhanced sugar metabolism in Streptococcus mutans co-cultured with Candida albicans within mixedspecies biofilms. Front. Microbiol. 8, 1036. https://doi.org/10.3389/fmicb.2017.01036 (2017).

22. Human Microbiome Project Consortium. Structure, function and diversity of the healthy human microbiome. Nature 486, $207-214$. https://doi.org/10.1038/nature11234 (2012).

23. Bernardi, S. et al. Combining culture and culture-independent methods reveals new microbial composition of halitosis patients' tongue biofilm. Microbiologyopen 9, e958. https://doi.org/10.1002/mbo3.958 (2020).

24. Richards, V. P. et al. Phylogenomics and the dynamic genome evolution of the genus Streptococcus. Genome Biol. Evol. 6, 741-753. https://doi.org/10.1093/gbe/evu048 (2014).

25. Abranches, J. et al. Biology of oral streptococci. Microbiol. Spectr. https://doi.org/10.1128/microbiolspec.GPP3-0042-2018 (2018).

26. Duan, D., Scoffield, J. A., Zhou, X. \& Wu, H. Fine-tuned production of hydrogen peroxide promotes biofilm formation of Streptococcus parasanguinis by a pathogenic cohabitant Aggregatibacter actinomycetemcomitans. Environ. Microbiol. 18, 4023-4036. https://doi.org/10.1111/1462-2920.13425 (2016).

27. Geng, J. et al. Complete genome and transcriptomes of Streptococcus parasanguinis FW213: Phylogenic relations and potential virulence mechanisms. PLoS ONE 7, e34769. https://doi.org/10.1371/journal.pone.0034769 (2012).

28. Kreth, J., Zhang, Y. \& Herzberg, M. C. Streptococcal antagonism in oral biofilms: Streptococcus sanguinis and Streptococcus gordonii interference with Streptococcus mutans. J. Bacteriol. 190, 4632-4640. https://doi.org/10.1128/JB.00276-08 (2008).

29. Redanz, S. et al. Pyruvate secretion by oral streptococci modulates hydrogen peroxide dependent antagonism. ISME J. 14, 10741088. https://doi.org/10.1038/s41396-020-0592-8 (2020).

30. Diaz, P. I. et al. Synergistic interaction between Candida albicans and commensal oral streptococci in a novel in vitro mucosal model. Infect. Immun. 80, 620-632. https://doi.org/10.1128/IAI.05896-11 (2012).

31. Lobo, C. I. V. et al. Dual-species biofilms of Streptococcus mutans and Candida albicans exhibit more biomass and are mutually beneficial compared with single-species biofilms. J. Oral. Microbiol. 11, 1581520. https://doi.org/10.1080/20002297.2019.15815 20 (2019).

32. Herrero, E. R. et al. Antimicrobial effects of commensal oral species are regulated by environmental factors. J. Dent. 47, 23-33. https://doi.org/10.1016/j.jdent.2016.02.007 (2016).

33. Zheng, L. Y., Itzek, A., Chen, Z. Y. \& Kreth, J. Oxygen dependent pyruvate oxidase expression and production in Streptococcus sanguinis. Int. J. Oral. Sci. 3, 82-89. https://doi.org/10.4248/IJOS11030 (2011).

34. Scoffield, J. A. \& Wu, H. Oral streptococci and nitrite-mediated interference of Pseudomonas aeruginosa. Infect. Immun. 83, 101-107. https://doi.org/10.1128/IAI.02396-14 (2015).

35. Kreth, J., Merritt, J., Shi, W. \& Qi, F. Competition and coexistence between Streptococcus mutans and Streptococcus sanguinis in the dental biofilm. J. Bacteriol. 187, 7193-7203. https://doi.org/10.1128/JB.187.21.7193-7203.2005 (2005).

36. Garcia-Mendoza, A., Liebana, J., Castillo, A. M., de la Higuera, A. \& Piedrola, G. Evaluation of the capacity of oral streptococci to produce hydrogen peroxide. J. Med. Microbiol. 39, 434-439. https://doi.org/10.1099/00222615-39-6-434 (1993).

37. Xiao, J. et al. The exopolysaccharide matrix modulates the interaction between 3D architecture and virulence of a mixed-species oral biofilm. PLoS Pathog. 8, e1002623. https://doi.org/10.1371/journal.ppat.1002623 (2012).

38. Alem, M. A., Oteef, M. D., Flowers, T. H. \& Douglas, L. J. Production of tyrosol by Candida albicans biofilms and its role in quorum sensing and biofilm development. Eukaryot. Cell 5, 1770-1779. https://doi.org/10.1128/EC.00219-06 (2006).

39. Desai, J. V. et al. Regulatory role of glycerol in Candida albicans biofilm formation. MBio 4, e00637-e00612. https://doi.org/10.1128/ mBio.00637-12 (2013).

40. Koo, H., Xiao, J., Klein, M. I. \& Jeon, J. G. Exopolysaccharides produced by Streptococcus mutans glucosyltransferases modulate the establishment of microcolonies within multispecies biofilms. J. Bacteriol. 192, 3024-3032. https://doi.org/10.1128/JB.01649 -09 (2010).

41. Jenkinson, H. F., Lala, H. C. \& Shepherd, M. G. Coaggregation of Streptococcus sanguis and other streptococci with Candida albicans. Infect. Immun. 58, 1429-1436. https://doi.org/10.1128/IAI.58.5.1429-1436.1990 (1990).

42. Scoffield, J. et al. Dietary nitrite drives disease outcomes in oral polymicrobial infections. J. Dent. Res. 98, 1020-1026. https://doi. org/10.1177/0022034519855348 (2019).

43. Sztajer, H. et al. Cross-feeding and interkingdom communication in dual-species biofilms of Streptococcus mutans and Candida albicans. ISME J. 8, 2256-2271. https://doi.org/10.1038/ismej.2014.73 (2014).

44. Scoffield, J. A., Duan, D., Zhu, F. \& Wu, H. A commensal streptococcus hijacks a Pseudomonas aeruginosa exopolysaccharide to promote biofilm formation. PLoS Pathog. 13, e1006300. https://doi.org/10.1371/journal.ppat.1006300 (2017).

45. Zhang, Q. et al. Structure-based discovery of small molecule inhibitors of cariogenic virulence. Sci. Rep. 7, 5974. https://doi. org/10.1038/s41598-017-06168-1 (2017).

46. Ren, Z. et al. Molecule targeting glucosyltransferase inhibits Streptococcus mutans biofilm formation and virulence. Antimicrob. Agents Chemother. 60, 126-135. https://doi.org/10.1128/AAC.00919-15 (2016).

47. Nijampatnam, B. et al. Inhibition of Streptococcus mutans biofilms by the natural stilbene piceatannol through the inhibition of glucosyltransferases. ACS Omega 3, 8378-8385. https://doi.org/10.1021/acsomega.8b00367 (2018). 
48. Filkins, L. M. et al. Prevalence of streptococci and increased polymicrobial diversity associated with cystic fibrosis patient stability. J. Bacteriol. 194, 4709-4717. https://doi.org/10.1128/JB.00566-12 (2012).

49. O’Toole, G. A. Microtiter dish biofilm formation assay. J. Vis. Exp. https://doi.org/10.3791/2437 (2011).

\section{Acknowledgements}

We thank Shawn Williams at the University of Alabama at Birmingham High Resolution Imaging Facility for his assistance with the Nikon A1 Confocal microscope and imaging analysis. This project was supported by the following funds and grants awarded to J.A.S: the University of Alabama at Birmingham Department of Microbiology startup funds, National Institutes of Health/National Institute of Dental and Craniofacial Research Grant R00 DE025913, and the American Association for Dental Research/Proctor and Gamble Underrepresented Faculty Research Fellowship.

\section{Author contributions}

J.H. and J.S. conceived and performed the experiments and analyzed the results and co-wrote the paper. Both authors discussed the results and revised the manuscript.

\section{Competing interests}

The authors declare no competing interests.

\section{Additional information}

Supplementary information is available for this paper at https://doi.org/10.1038/s41598-020-76744-5.

Correspondence and requests for materials should be addressed to J.A.S.

Reprints and permissions information is available at www.nature.com/reprints.

Publisher's note Springer Nature remains neutral with regard to jurisdictional claims in published maps and institutional affiliations.

(c) (i) Open Access This article is licensed under a Creative Commons Attribution 4.0 International License, which permits use, sharing, adaptation, distribution and reproduction in any medium or format, as long as you give appropriate credit to the original author(s) and the source, provide a link to the Creative Commons licence, and indicate if changes were made. The images or other third party material in this article are included in the article's Creative Commons licence, unless indicated otherwise in a credit line to the material. If material is not included in the article's Creative Commons licence and your intended use is not permitted by statutory regulation or exceeds the permitted use, you will need to obtain permission directly from the copyright holder. To view a copy of this licence, visit http://creativecommons.org/licenses/by/4.0/.

(C) The Author(s) 2020 